\section{Cause of Changes in Rate of some Gas Reactions}

IN 1932 Travers and Hockin described a phenomenon associated with the formation of methane and of condensation products during the pyrolysis of pure ethane ${ }^{1}$. When this gas is heated to the neighbourhood of $600^{\circ}$, ethylene and hydrogen are formed very rapidly, and a state is approached rapidly approximating to equilibrium in the ethane-ethylenehydrogen system. Methane and condensation products are also formed, and as Travers and Pearce show in a paper which will shortly appear in the Transactions of the Society of Chemical Industry, both processes originate independently from the ethylene. When the pyrolysis has proceeded for a short time, the rates of formation of methane and of condensate suddenly and simultaneously slow down. However, if instead of using pure ethane, a mixture of ethane, ethylene and hydrogen in equilibrium proportions is used, no breaks are observed in the graphs representing the rates of formation of methane and condensate at all. The initial rate of both processes is definitely greater when starting from pure ethane than when starting from an equilibrium mixture, and while in the former case the processes are markedly affected by surface, in the latter the effect of surface appears to be negligible.

In the paper now in the press, Travers and Pearce explain the phenomenon in the following way. The energy of activation of the primary decomposition of ethane into ethylene and hydrogen is about $76 \mathrm{k.cal}$. while the energy requirements of the process are about $31 \mathrm{k.cal}$. As a consequence, the energy to be disposed of is $45 \mathrm{k}$.cal. The result is that, superimposed upon the process by which methane and condensate are formed in equilibrium mixtures, there is, in the case of the initial stages of the pyrolysis of pure ethane, an energy chain mechanism, which continues up to a point which is possibly determined by the formation of condensation products of high molecular weight, of which the rate is determined by surface conditions. However, the cause of the whole phenomenon is to be found in the existence of the reaction involving the primary decomposition of the ethane. This is what is referred to in this laboratory as a background process, and on such background processes identical phenomena in the case of other reactions appear to depend.

In the course of the discussion at the Royal Society on May 10, we described some experiments which were being carried out in this laboratory on the thermal decomposition of acetaldehyde, which indicated that a similar phenomenon occurred in this case. Attention was directed to the fact, because it seemed to have some bearing on a phenomenon observed by $\mathrm{Mr}$. Hinshelwood ${ }^{2}$ in an investigation on the thermal decomposition of the same compound, to which he has made reference in several papers. Mr. Hinshelwood, in commenting on our note ${ }^{3}$, deniod that there was any connexion between our observations and his own, but we find it difficult to see wherein the difference lies. However, we feel very sure that the following facts cannot be ignored.

One cannot consider the pyrolysis of acetaldehyde without taking careful account of the experimental work of Prof. W. A. Bone ${ }^{4}$ on the occurrence of this compound and its isomers, among the products of oxidation of ethylene, or avoid the possibility that internal change such as,

$$
\text { Acetaldehyde = Vinyl alcohol, }
$$

may constitute a background reaction such as operates in the early stages of the pyrolysis of pure ethane. In this case, the system may be a much more complex one than that which we have to consider in the case of ethane, which may account for the facts put forward in our contribution to the discussion of May 10. It does not seem to be at all unlikely that the formation of methane and carbon monoxide from acetaldehyde involves the previous formation of vinyl alcohol, and that the mechanism of the subsequent reaction is similar to that which Travers and Pearce have suggested for the formation of methane and condensation products from pure ethane.

In the case of the thermal decomposition of dimethyl ether, we have a process operating in two stages. In the first stage methane and formaldehyde are formed, the rate of the reaction being measured by the rate of formation of methane. There are no breaks in the graphs representing this process, which is in accord with the view which is now put forward, for there is no obvious background process. The second stage, the decomposition of the formaldehyde, which can be followed separately when using our analytical method of investigation, exhibits the phenomenon which we have described. The background process is most probably the process of primary decomposition of the dimethyl ether.

Finally, we must refer to an investigation on the hydrogenation of diphenyl to benzene which was carried out in this laboratory by Dr. J. E. Sisson. In this case, breaks which occur in the graphs representing the rate of formation of benzene appear to be related to maxima in the concentra. tion of diphenyl-benzene, which is formed during the process of hydrogenation by a process which does not appear to be a simple one, and may act as a background reaction to the hydrogenation process.

It seems possible that reactions involving the thermal decomposition of organic substances fall into two classes. In one of these, in the early stages, an energy chain system is imposed upon a simpler process, the energy chains being initiated by some background process, tending towards equilibrium in the system. In the other class such a background process does not operate, and the main process is of a simpler character. However, even in such processes the products of reaction may still influence activation, as indicated in our contribution to the discussion on May 10, and developed in the paper by Travers and Pearce to which reference has been made.

We are continuing the investigations on acetaldehyde and dimethyl ether by the method of detailed analysis which has been described elsewhere. Though exact, the method is laborious and the work takes a long time.

MorRIS W. Travers.

Robert V. SEDdon. Peter F. Gay.

Department of Chemistry,

The University,

Bristol.

Sept. 29.

\footnotetext{
${ }^{2}$ Proc. Roy. Soc., A, 136, 1 ; 1932.

- NAture, 131, 24, Jan. 7, 1933.

'Proc. Roy. Soc., A, 146, 252 ; 1934.

4 Proc. Roy. Soc., A, 143, 16 ; 1933.
} 